\title{
Cost analysis of pulmonary lobectomy procedure: comparison of stapler versus precision dissection and sealant
}

This article was published in the following Dove Press journal:

ClinicoEconomics and Outcomes Research

3 April 2017

Number of times this article has been viewed

\author{
Andrea Droghetti ${ }^{1}$ \\ Giuseppe Marulli² \\ Jacopo Vannucci ${ }^{3}$ \\ Michele Giovanardi' \\ Maria Caterina Bottoli' \\ Mark Ragusa ${ }^{3}$ \\ Giovanni Muriana' \\ 'Thoracic Surgery Division, Carlo \\ Poma Hospital, Mantova, ${ }^{2}$ Thoracic \\ Surgery Division, University of \\ Padova, Padova, ${ }^{3}$ Thoracic Surgery \\ Division, University of Perugia, \\ Perugia, Italy
}

Objective: We aimed to evaluate the direct costs of pulmonary lobectomy hospitalization, comparing surgical techniques for the division of interlobar fissures: stapler (ST) versus electrocautery and hemostatic sealant patch (ES).

Methods: The cost comparison analysis was based on the clinical pathway and drawn up by collecting the information available from the Thoracic Surgery Division medical team at Mantova Hospital. Direct resource consumption was derived from a previous randomized controlled trial including 40 patients. Use and maintenance of technology, equipment and operating room; administrative plus general costs; and 30-day use of postsurgery hospital resources were considered. The analysis was conducted from the hospital perspective.

Results: On the average, a patient submitted to pulmonary lobectomy costs $€ 9,744.29$. This sum could vary from $€ 9,027$ (using ES) to $€ 10,460$ (using ST). The overall lower incidence ( $50 \%$ vs $95 \%, P=0.0001)$ and duration of air leakage ( 1.7 days vs 4.5 days, $P=0.0001)$ in the ES group significantly affects the mean time of hospital stay (11.0 days vs 14.3 days) and costs. Cost saving in the ES group was also driven by the lower incidence of complications. The main key cost driver was staff employment (42\%), then consumables (34\%) and operating room costs (12\%). Conclusion: There is an overall saving of around $€ 1,432.90$ when using ES patch for each pulmonary lobectomy. Among patients undergoing this surgical procedure, ES can significantly reduce air leakage incidence and duration, as well as decrease hospitalization rates. However, further multicenter research should be developed considering different clinical and managerial settings. Keywords: pulmonary lobectomy, hemostatic sealant patch, TachoSil ${ }^{\mathbb{R}}$, economic evaluation, cost analysis

\section{Introduction}

Lung cancer is a very common malignancy with the peculiar characteristic of high aggressivity and lethality, so it has been called the "big killer". Pulmonary lobectomy is the operation of choice for the standard treatment of early-stage lung cancer. It encompasses an approach through the pleural cavity and the ligation of the pulmonary artery branches, the pulmonary vein and the bronchus. It is usually considered as the removal of half of the lung.

Although a few studies have supported the evidence of favorable efficacy of fleecebound sealing, very few trials are aimed at cost analyses. A couple of investigations have been conducted to evaluate health care costs after a pulmonary lobectomy. These analyses have demonstrated that an overall saving of around $€ 100-200$ is achieved if fleece-bound sealing procedure is performed instead of the standard techniques of sealing the lung following pulmonary resection. ${ }^{1,2}$ 
Nonetheless, no detailed data on the economic impact of fleece-bound sealing during pulmonary lobectomy procedure are currently available, and the incidence of prolonged air leak is variable in the data in the literature. Considering the results from the clinical trial carried out in an Italian Hospital, this study was designed with the aim to give better insight into the definite hospital direct costs by using an activity-based costing approach. ${ }^{2}$ Costs occurring during hospitalization for alternative surgical techniques to seal interlobar fissures division were analyzed. Stapler (ST) versus electrocautery and hemostatic sealant patch (ES) procedures were performed at the same Italian Hospital and economically compared.

As economical benefit has been reported in the clinical trial, further evaluation of this aspect has been considered a potentially interesting subject to be evaluated in order to clarify the impact on costs of clinical behavior and thus to help physicians perform a safe and sustainable surgery.

\section{Patients and methods}

The analysis focuses on the comparative assessment of hospitalization-related direct costs for patients undergoing pulmonary lobectomy using two different surgical management techniques (ST versus ES) to achieve the interlobar fissure dissection. The study was carried out at the Carlo Poma Hospital of Mantova by the Thoracic Surgery medical team, and the cost analysis of both techniques was based on specific economic data collection on the patient population (40 patients, 20 in each group) already enrolled for the previous trial (prospective, randomized), which compared the clinical outcome comparing ST and ES (Table 1). ${ }^{2}$ Use and maintenance of equipment, technology and operating room; the general plus administrative costs; and hospital resources used in the postoperative 30-day period as outpatients, entered the analysis. The total cost for each patient

Table I Resource consumption

\begin{tabular}{lll}
\hline Variables & $\begin{array}{l}\text { Stapler } \\
\text { group (ST) }\end{array}$ & $\begin{array}{l}\text { Electrocautery and } \\
\text { sealant group (ES) }\end{array}$ \\
\hline Length of stay, days & 14.3 & $1 \mathrm{I}$ \\
Operating room time, minutes & 189.8 & 188.5 \\
Gender (male), \% & 55 & 90 \\
Residual lung cavity incidence, \% & 40 & 5 \\
Days with residual lung cavity & 2 & 2 \\
Air leakage incidence, \% & 95 & 50 \\
Days with air leaks & 3.7 & 1.7 \\
Mean number of chest & 5.35 & 5.75 \\
radiographs & & \\
Mean number of stapler & 1.5 & 1.4 \\
recharge/patches used per & & \\
patient & & \\
\hline
\end{tabular}

Note: Data from Droghetti et al. ${ }^{2}$ was calculated considering the whole medical process, taken from hospital charts, from the day of first admission at the medical center to the medical evaluation on the postoperative 30th day.

The analysis was conducted from the hospital perspective. The databases used did not contain a variable allowing for the individual identification of patients. According to Italian legislation, this study's characteristics (observational methodology and retrospective analysis) do not make it necessary to obtain authorization from the hospital ethics committee. In accordance with Italian privacy law (code concerning the protection of personal data, 30 June 2003, n.196) patients were assigned identification numbers for the study, thus eliminating the patient health service codes and avoiding the risk of identifying patients personally. A previous clinical trial $^{2}$ showed interesting data regarding cost-effectiveness, but the analysis was insufficient to give an accurate sense of the potential economical impact of the different surgical approaches.

\section{Surgery}

Two groups of patients (20 patients each) were subjected to pulmonary lobectomy. The interlobar fissure was approached by different techniques. In one group, the fissure was divided by means of mechanical STs, while in the second group, this passage was accomplished using electrocautery for dissection and collagen patches coated with human fibrinogen and thrombin $\left(\mathrm{TachoSi}^{\circledR}\right)$ for sealing. Lobectomy was performed in the same way for the remaining steps of the procedure.

\section{Resource consumption and cost analysis}

Data on material consumption and resource management were analyzed by hospital representatives who are involved in the early-stage non-small cell lung cancer (NSCLC) patient pathway from workup to surgery. For all countable consumables and equipment exploitation in terms of time of use and maintenance, in addition to administrative, general and human resource service, costs were estimated.

Costs per unit were collected directly from hospital accounts where possible, from regional tariffs for specialized services and from hospital admissions where data were not available from the hospital (Table 2). Episodes of complications and comorbidities were identified as well, including related resource consumption, and their occurrence was referred to in a previous clinical trial. ${ }^{2}$ A differential analysis was carried out to identify the variation between using ST versus ES in terms of resource consumption. All costs were updated to the year 2012. ${ }^{3-5}$ 
Table 2 Unit cost sources

\begin{tabular}{ll}
\hline Resources & Sources \\
\hline Hospital staff cost per hour & Hospital accounts \\
Consumables & Hospital accounts \\
Drug & AlFA, Lista di trasparenza 20I2 \\
Diagnostic examinations & Regional tariffs for Specialist \\
Technology equipment amortization & Services (2012) \\
Operating room & Hospital accounts \\
Administrative and general expenses* & Hospital accounts \\
\hline
\end{tabular}

Note: *Includes patient treatment service costs.

Abbreviation: AIFA, Italian Medicines Agency (L'Agenzia Italiana del farmaco).

\section{Sensitivity analysis}

Single-way sensitivity analysis was performed by varying $( \pm 10 \%)$ the cost of hemostatic sealant patch to establish the robustness of the model. ${ }^{6,7}$ Because the difference in mean hospitalization length of the two procedures did not achieve statistical significance, a sensitivity analysis technique was additionally applied to determine the economic value assigned to the ST scenario versus the ES scenario assuming the same length of stay (LOS) for both the situations. ${ }^{6}$

\section{Results}

The hospitalization cost for the pulmonary lobectomy procedure totaled around $€ 9,027.95-10,460.64$ and it diverged if surgical ST or ES had been used to complete the interlobar fissures. The amount varied from $€ 9,027$ for the ES group to $€ 10,460$ for the ST group. The main cost driver was recognized to be the staff time (around 20\%), with the consumables (including both STs and patches, around 19\%) being the second (Table 3 ). Resource consumption and the consequential costs showed that the difference between the two scenarios, excluding overheads, was mainly due to time spent by involved staff (ES staff cost was $10.2 \%$ lower than the staff cost for STs) and drugs used to treat both expected consequences of lobectomy and complications (-20.6\%) (Table 3$)$. Indeed, the lower overall incidence (50\% vs $95 \%$, $P=0.0001)$ and duration of air leakage (1.7 days vs 4.5 days, $P=0.0001)$ in the ES group significantly affected treatment of complications and consequently the mean time to hospital discharge (11.0 days vs 14.3 days) and ultimately the costs (Table 3 and Figure 1 ). ${ }^{2}$ A sensitivity analysis was performed to determine whether variations in the cost of the hemostatic

Table 3 Results: mean cost per resource type

\begin{tabular}{llllll}
\hline Variables & Stapler group (ST) & $\%$ & Electrocautery and sealant group (ES) & \% & Difference, ES vs ST, \% \\
\hline Staff time & $€ 2,050.44$ & 19.6 & $€ 1,840.60$ & 20.4 & -10.2 \\
Diagnostic tests & $€ 315.30$ & 3.0 & $€ 322.27$ & 3.6 & 2.2 \\
Drugs & $€ 349.70$ & 3.3 & $€ 277.62$ & 3.1 & -20.6 \\
Consumables & $€ 1,893.22$ & 18.1 & $€ 1,738.24$ & 19.3 & -8.2 \\
Overheads & $€ 5,851.98$ & 55.9 & $€ 4,849.22$ & 53.7 & -17.1 \\
Total hospitalization cost & $€ 10,460.64$ & & $€ 9,027.95$ & -13.7 & \\
\hline
\end{tabular}

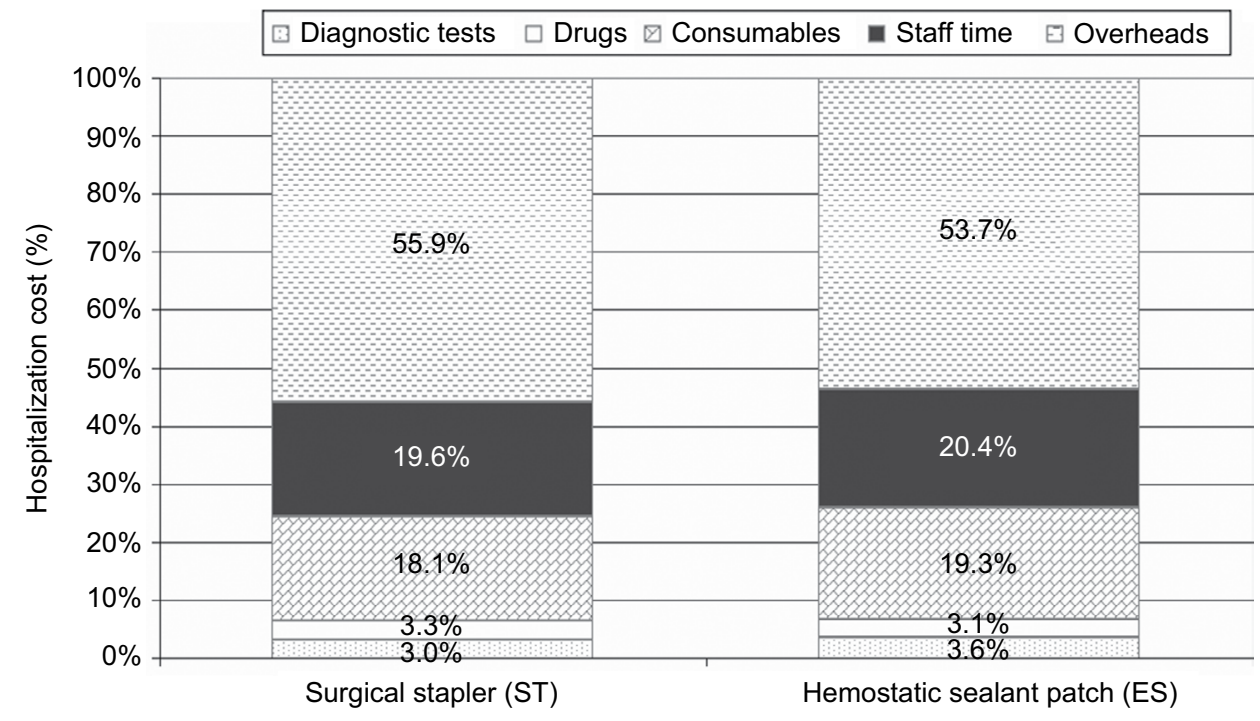

Figure I Results: mean cost of procedure. 
sealant patch $( \pm 10 \%)$ could influence the results of the cost analysis investigation considering possible regional variation of price. Therefore, the cost of the hemostatic sealant patch was varied $( \pm 10 \%)$ to determine the circumstances that could logically bring about cost change.

Results basically remained unremarkable, varying from $€ 8,978$ ( $-10 \%$ cost) to $€ 9,077$ ( $+10 \%$ cost $)$. In addition, even when the hospital LOS for the ST group was decreased to 11 days (the same situation as that for the ES procedure), the average hospitalization cost using the ES technique was less expensive than that with ST (€9,341 vs €9,028). Higher costs of the ST group depend on consumables and therapies for complications (with higher incidence in ST group vs ES group). Consequently, it could be stated that even if LOS is assumed to be equal between the two groups, ES treatment remained less expensive. No readmission occurred within the 30-day follow-up time.

\section{Discussion}

The most commonly performed procedure in the treatment of early-stage NSCLC is pulmonary lobectomy, which currently represents the standard of cure. ${ }^{8}$ Surgery is considered the preeminent therapeutic option. ${ }^{9-11}$ Complications after lung manipulation are often related to the stressed residual parenchyma and may heavily affect the postoperative course of patients.

One of the important events potentially influencing the healing process after major surgery is persistent air leak from lung parenchyma. This phenomenon is reported to appear in $3 \%-25 \%$ of patients. ${ }^{12}$ The leakage showed higher incidence when lung fissures were not complete, and it could persist for several days. Surgical dissection of fissure to anatomically divide the two lobes generally is causative of this prolonged air leakage, which requires long-lasting chest drainage. This clinical circumstance hampers recovery and limits hospital discharge. ${ }^{13-15}$ Hence, the negative impact of postoperative morbidity expands the hospital stay period with consequences on the economic aspect.

Over the years, there has been consistent progress in terms of surgical devices and medical products so that surgeons have a spectrum of choices in order to perform each surgical procedure. The panel of options includes STs, traditional suture materials, energy devices or electrocautery unit, in addition to fibrin glue, synthetic polyethylene glycol-based materials and collagen patches coated with fibrinogen and thrombin (TachoSil ${ }^{\circledR}$; Takeda Pharmaceutical Company, Osaka, Japan). ${ }^{14,16-21}$ Several authors have investigated the topic of fleece-bound sealing with emphasis on efficacy and economical advantage. ${ }^{14,15,17}$ These studies are international and one is a prospective randomized study performed by a single center; ${ }^{16}$ these contributions have brought to evidence a significantly lower consistency of the air leakage phenomenon in the early postoperative period when the technique of fleece-bound sealing is performed; consequently early chest tube removal and a shorter hospitalization are reported. ${ }^{15,16}$

A prospective randomized controlled trial has been performed by an Italian research group to evaluate the comparative outcome of performing the fissure dissection by means of alternative procedures.

Results of such a study were consistent with other international analyses and, significantly greater incidence and severity of intraoperative air leaks were observed in the ES group and a relevant reduction of these on postoperative day 1 after application of TachoSil. ${ }^{17}$ The use of ES patch (TachoSil) for aerostasis to achieve surgical dissection of pulmonary fissures to accomplish a lobectomy resulted in an overall saving of approximately $€ 1,432.69$ compared to the control group. Among all the enrolled patients undergoing surgery, ES was significantly related to reduced incidence of air leakage and duration of such leakage, as well as the in-hospital LOS. This evidence could be highly remarkable considering specific economic arrangements of expense reimbursement, such as the Diagnosis Related Groups system. This may mean that, in situations in which health care providers need to achieve the therapeutic goal besides guaranteeing the optimized use of resources such as as-short-as-possible stay in hospital, the provided clinical services must be at the state-of-the-art but must simultaneously tend to the highest possible level of technical quality, to facilitate the best outcome for the patient along with the best economic management.

These results are comparable with conclusions of other studies as, eg, a recent systematic review summarizing the available evidence for fleece-bound sealing. ${ }^{14-18,20,22}$ The review analyzed 49 publications covering different surgical sectors, with the conclusion that, in addition to clinical benefits, fleece-bound sealing resulted in economic benefits also, such as reduction in time to hospital discharge and complication rates. ${ }^{15}$ Moreover, the efficacy of fleece-bound tissue gluing to seal air leaks after pulmonary resection and primary leakage closure was evaluated in a prospective, randomized, open-label, parallel-group, single-center study for NSCLC surgery. ${ }^{16}$ The aim of such a study was to compare fleece-bound sealing (TachoSil, TS) and standard techniques (ST, defined as suture with or without staples) for closure of alveolar air leaks following parenchymal resection. Significant reductions in volume of air leakage in the early postoperative period, with early chest drain removal and time to hospital discharge, were demonstrated after using 
fleece-bound sealing technique in the subset of patients with recognized intraoperative air leakage. ${ }^{16}$

The clinical trial by Droghetti et $\mathrm{al}^{2}$ highlighted the benefit of TachoSil patching, which could lead to a reduction of $>48$ hours in the mean time to chest drain removal and $>3$ days of hospital stay. Multiple aspects of possible superiority of the use of fleece-bound sealants resulted from the ES group outcome. Among these features, the ability to perform an accurate dissection to preserve as much pulmonary parenchyma as possible allows the residual lung to be larger and to preserve more elasticity, which entails enhanced propensity to refill the thoracic cavity, than after being stapled. ${ }^{17}$ Such a condition leads to a minor incidence of complications and, with the present cost analysis, it was demonstrated that these benefits also imply cost saving for the health care system.

Despite the quite different results, more specifically concerning total amounts, the evidence produced by this analysis confirmed what was inferred from other economic evaluations carried out in Europe. ${ }^{6,23}$ These investigations demonstrated how the use of fleece-bound sealants to stop air leakage could reduce the length of hospital stay and what effect this may have on economic impact. In another study, the number and frequency of persistent leakages were also found to be reduced after fleece-bound sealing, but the duration of hospitalization was not examined and no economic comment was given. ${ }^{14}$ This study has several limitations. The study was performed in a single-center setting and the population included in the study was small. So, several factors could have had some impact in producing biases considering the specific unique environment of a single institution. The number of patients included in the study is limited and this represents a limitation for the investigation. However, the data strongly suggest that using fleece-bound sealing to treat and prevent air leakage after lobectomy is worthwhile from the clinical point of view and from the hospital economic perspective as well.

In the context of local and general previous analyses on cost-effectiveness, this study represents a further experience, but it remains unclear how important the impact could be on cost-effectiveness due to local and national regulations and type of supplies.

\section{Conclusion}

In the future, our ability to identify which topical hemostatic agent or surgical sealant should be used in a particular patient will depend increasingly on the quality of information available regarding prevention of postoperative complications in clinical practice and, indirectly, on the possibility of optimizing the use of hospital services. Considering that the study has been performed from the hospital perspective, some specific clinicoeconomical aspects could be delineated to arrange management of materials for a more sustainable and appropriate use of instruments in clinical practice. Highquality information would be necessary to optimize total health expenditure and simultaneously improve patients' quality of life. From a historical point of view, the analysis of benefits from using new tools and materials in clinical practice is increasingly necessary for resource planning in the future of surgical specialties. This economical and managerial necessity is testified by the increasing number of references (clinical and experimental) supporting innovative, effective and safe use of sealants for different new indications. ${ }^{24-28}$ However, further multicenter research in different clinical and organizational settings would be necessary in order to implement and validate these results.

\section{Acknowledgments}

This research was funded by an unrestricted grant from Takeda Italia Spa. We are grateful for the collaboration and support of the Administration of Carlo Poma Hospital, Italy.

\section{Disclosure}

The authors are employees of independent research organizations and have maintained independent scientific control over the study, including data analysis and interpretation of final results. The authors report no other conflicts of interest in this work.

\section{References}

1. Anegg U, Rychlik R, Smolle-Jüttner F. Do the benefits of shorter hospital stay associated with the use of fleece-bound sealing outweigh the cost of the materials. Interact Cardiovasc Thorac Surg. 2008;7: 292-296.

2. Droghetti A, Schiavini A, Muriana P, et al. A prospective randomized trial comparing completion technique of fissures for lobectomy: stapler versus precision dissection and sealant. J Thorac Cardiovasc Surg. 2008;136(2):383-391.

3. Italian Health Economics Association-Associazione Italiana di Economia Sanitaria (AIES). Italian Guidelines proposal on how to conduct economic evaluation studies of health programs. Pharmacoeconomics. 2009;11:83-93.

4. Agenzia Italiana del Farmaco [AIFA; Italian Drug Agency] [webpage on the Internet]. Negoziazione e rimborsabilitá [Negotiation and reimbursement; web page on the Internet]. Rome: AIFA; 2013. Italian. Available from: http://www.agenziafarmaco.gov.it/it/content/negoziazione-erimborsabilit\%C3\%A0. Accessed November 13, 2011.

5. Istat [webpage on the Internet]. Indice dei prezzi al consumo per le rivalutazioni monetarie [Consumer Price Index for inflation adjustments]. Roma: Istituto nazionale di statistica. Available from: http:// www.istat.it/it/archivio/30440. Accessed November 7, 2013.

6. Briggs A, Sculpher M, Buxton M. Uncertainty in the economic evaluation of health care technologies: the role of sensitivity analysis. Health Econ. 1994;3:95-104. 
7. Husereau D, Drummond M, Petrou S, et al; ISPOR Health Economic Evaluation Publication Guidelines-CHEERS Good Reporting Practices Task Force. Consolidated health economic evaluation reporting standards (CHEERS) - explanation and elaboration: a report of the ISPOR Health Economic Evaluation Publication Guidelines Good Reporting Practices Task Force. Value Health. 2013;16(2):231-250.

8. Mezzetti M, Panigalli T, Giuliani L, Raveglia F, Giudice FL, Meda S. Personal experience in lung cancer sleeve lobectomy and sleeve pneumonectomy. Ann Thorac Surg. 2002;73(6):1736-1739.

9. Damstrup L, Poulson HS. Review of the curative role of radiotherapy in the treatment of non-small cell lung cancer. Lung Cancer. 1994; 11(3-4):153-178.

10. Grili R, Oxman AD, Julian JA. Chemotherapy for advanced non-small cell lung cancer; how much benefit with enough? J Clin Oncol. 1993; 11:1855-1872.

11. Rena O, Papalia E, Mineo TC, et al. Air-leak management after upper lobectomy in patients with fused fissure and chronic obstructive pulmonary disease: a pilot trial comparing sealant and standard treatment. Interact Cardiovasc Thorac Surg. 2009;9(6):973-977.

12. Droghetti A, Schiavini A, Muriana P, et al. Autologous blood patch in persistent air leaks after pulmonary resection. $J$ Thorac Cardiovasc Surg. 2006;132(3):556-559.

13. Stolz AJ, Schutzner J, Lischke R, Simonek J, Pafko P. Predictors of prolonged air leak following pulmonary lobectomy. Eur J Cardiothorac Surg. 2005;27(2):334-336.

14. Wain JC, Kaiser LR, Johnstone DW, et al. Trial of a novel synthetic sealant in preventing air leaks after lobectomy. Ann Thorac Surg. 2001; 71:1623-1629.

15. Serra-Mitjans M, Belda-Sanchis J, Rami-Porta R. Surgical sealant for preventing air leaks after pulmonary resections in patients with lung cancer. Cochrane Database Syst Rev. 2005;20(3):CD003051. doi: 10.1002/14651858.CD003051.pub2.

16. Fabian T, Federico JA, Ponn RB. Fibrin glue in pulmonary resection: a prospective randomized blinded study. Ann Thorac Surg. 2003;75(5): 1587-1592.

17. Fleisher AG, Evans KG, Nelems B, Finley RJ. Effect of routine fibrin glue use on the duration of air leaks after lobectomy. Ann Thorac Surg. 1990;49(1):133-134.
18. Wong K, Goldstraw P. Effect of fibrin glue in the reduction of postthoracotomy alveolar air leak. Ann Thorac Surg. 1997;64(4): 979-981.

19. Macchiarini P, Wain J, Almy S, Dartevelle P. Experimental and clinical evaluation of a new synthetic, absorbable sealant to reduce air leaks in thoracic operations. J Thorac Cardiovasc Surg. 1999;117(4):751-758.

20. Porte HL, Jany T, Akkad R, et al. Randomized controlled trial of a synthetic sealant for preventing alveolar air leaks after lobectomy. Ann Thorac Surg. 2001;71(5):1618-1622.

21. Lang G, Csekeo A, Stamatis G, et al. Efficacy and safety of topical application of human fibrinogenythrombin-coated collagen patch (TachoComb) for treatment of air leakage after standard lobectomy. Eur J Cardiothorac Surg. 2004;25:160-166.

22. Rychlik R. Zur Nutzenbewertung der Gewebeklebung in der Chirurgie-das Beispiel TachoSil [For the benefit assessment of tissue adhesion in the Surgery- the example TachoSil]. Krankenhauspharmazie. 2006;5:199-204. German.

23. Carbon RT, Baar S, Waldschmidt J, Hümmer HP, Simon S. Minimalinvasive Kinderchirurgie: Entwicklung und Fortschritt durch innovative Technologie. Die ATCS-Klebung [Minimal invasive paediatric surgery: further advances through innovative technology. ATCS sealing]. Klin Pädiatr. 2001;213:99-103. German.

24. Colombo GL, Bettoni D, Di Matteo S, et al. Economic and outcomes consequences of TachoSil ${ }^{\circledR}$ : a systematic review. Vasc Health Risk Manag. 2014;10:569-575.

25. Vannucci J, Droghetti A, Bufalari A, et al. Effectiveness and predictability of pleurodesis with the Tachosil ${ }^{\circledR}$ surgical patch compared with talc poudrage: an experimental study. Eur J Cardiothorac Surg. 2016; 50(4):668-674.

26. Giordano R, Palma G, Palumbo S, et al. Use of biological hemostatic support TachoSil ${ }^{\circledR}$ for reoperation in pediatric cardiac surgery. Minerva Pediatr. 2016;68(3):240-241.

27. Marano L, Di Martino N. Efficacy of human fibrinogen-thrombin patch (TachoSil) clinical application in upper gastrointestinal cancer surgery. J Invest Surg. 2016;29(6):352-358.

28. Feng W, Coady M. Epicardial Tachosil patch repair of ventricular rupture in a 90-year-old after mitral valve replacement. Ann Thorac Surg. 2016; 101(6):2361-2363.
ClinicoEconomics and Outcomes Research

\section{Publish your work in this journal}

ClinicoEconomics and Outcomes Research is an international, peerreviewed open-access journal focusing on health technology assessment, pharmacoeconomics and outcomes research in the areas of diagnosis, medical devices, and clinical, surgical and pharmacological intervention. The economic impact of health policy and health systems

\section{Dovepress}

organization also constitute important areas of coverage. The manuscript management system is completely online and includes a very quick and fair peer-review system, which is all easy to use. Visit http://www.dovepress.com/testimonials.php to read real quotes from published authors. 